\title{
Expanding Diversity in Molecular Structures and Functions of the IL-6/IL-12 Heterodimeric Cytokine Family
}

\author{
Hideaki Hasegawa, Izuru Mizoguchi, Yukino Chiba, Mio Ohashi, Mingli Xu \\ and Takayuki Yoshimoto*
}

Department of Immunoregulation, Institute of Medical Science, Tokyo Medical University, Tokyo, Japan

\section{OPEN ACCESS}

Edited by:

Masaaki Murakami, Hokkaido University, Japan

Reviewed by: Daisuke Kamimura, Hokkaido University, Japan Akihiro Kimura, National Center for Global Health and Medicine, Japan

*Correspondence: Takayuki Yoshimoto yoshimot@tokyo-med.ac.jp

Specialty section: This article was submitted to Inflammation, a section of the journal Frontiers in Immunology

Received: 30 July 2016 Accepted: 20 October 2016 Published: 04 November 2016

Citation: Hasegawa H, Mizoguchi I, Chiba Y, Ohashi M, Xu M and Yoshimoto T (2016) Expanding Diversity in Molecular Structures and Functions of the IL-6/IL-12 Heterodimeric Cytokine Family. Front. Immunol. 7:479. doi: 10.3389/fimmu.2016.00479
The interleukin (IL)-6/IL-12 family cytokines have pleiotropic functions and play critical roles in multiple immune responses. This cytokine family has very unique characteristics in that they comprise two distinct subunits forming a heterodimer and each cytokine and receptor subunit shares with each other. The members of this cytokine family are increasing; currently, there are more than six cytokines, including the tentatively named cytokines IL-Y (p28/p40), IL-12 (p35/p40), IL-23 (p19/p40), IL-27 [p28/Epstein-Barr virus-induced protein 3 (EBI3)], IL-35 (p35/EBI3), and IL-39 (p19/EBI3). This family of cytokines covers a very broad range of immune responses, including pro-inflammatory responses, such as helper T (Th)1, Th2, and Th17, to anti-inflammatory responses, such as regulatory $T$ (Treg) cells and IL-10-producing Treg cells. IL-12 is the first member of this family, and IL-12, IL-23, and IL-27 are mainly produced by activated antigenpresenting cells, such as dendritic cells and macrophages. IL-12 plays a critical role in the promotion of Th1 immune responses by inducing interferon- $\gamma$ production to combat pathogens and malignant tumors. IL-23 induces IL-17 production and is necessary to maintain pathogenic Th17 cells that cause inflammatory and autoimmune diseases. IL-27 was initially reported to play a critical role in promotion of Th1 differentiation; however, subsequent studies revealed that IL-27 has broader stimulatory and inhibitory roles by inducing IL-10-producing Treg cells. IL-35 is produced by forkhead box P3+ Treg cells and activated $B$ cells and has immunosuppressive functions to maintain immune tolerance. The most recently identified cytokine, IL-39, is produced by activated $\mathrm{B}$ cells and has pro-inflammatory functions. The cytokine tentatively named IL-Y seems to have anti-inflammatory functions by inhibiting Th1 and Th17 differentiation. In addition, individual cytokine subunits were also shown to have self-standing activities. Thus, promiscuity within the IL-6/IL-12 family cytokines complicates structural and functional clarification and assignment of individual cytokines. A better understanding of the recent advances and expanding diversity in molecular structures and functions of the IL-6/IL-12 family cytokines could allow the creation of novel therapeutic strategies by using them as tools and targeted molecules.

Keywords: IL-6, IL-12, IL-23, IL-27, IL-30, IL-35, IL-39, IL-Y

Abbreviations: CLF, cytokine-like factor 1; EBI3, Epstein-Barr virus-induced protein 3; gp130, glycoprotein 130; IFN, interferon; Ig, immunoglobulin; IL, interleukin; NK, natural killer; R, receptor; s, soluble; STAT, signal transducer and activator of transcription; Th, helper T; Treg, regulatory $\mathrm{T}$. 


\section{INTRODUCTION}

Generally, cytokines are grouped into distinct families depending on the differences in homology among amino acid sequences and structural characteristics. These characteristics include the protein higher-order structure and usage of certain membranebound cytokine $\beta$-receptors for signal transduction. In particular, the interleukin (IL)-6/IL-12 family cytokines have unique structure properties in that they comprise distinct $\alpha$-subunits and $\beta$-subunits forming a heterodimer and they share cytokine subunits and cellular receptors with each other (1-3). The $\alpha$-subunit is a four-helix bundle, long-chain structure similar to the type I cytokine, IL-6, and includes IL-23/IL-39p19, IL-27/ IL-30p28, and IL-12/IL-35p35. The $\beta$-subunit is composed of two tandem fibronectin type III domains that form a cytokinebinding homology region and an N-terminal immunoglobulin (Ig) domain. This subunit is structurally related to the nonsignaling receptor of IL- 6 , soluble IL- $6 \alpha$-receptor (sIL-6R $\alpha$ ), and includes IL-12/IL-23p40 and IL-27/IL-35/IL-39 Epstein-Barr virus-induced protein 3 (EBI3). By promiscuous pairing between these $\alpha$-subunits and $\beta$-subunits, more than six heterodimeric cytokines were reported to exist (Figure 1).

Interleukin-6 is one of the most important inflammatory cytokines; it is unique in signaling through not only membranebound IL-6R $\alpha$ but also sIL-6R $\alpha$ together with ubiquitously expressed $\beta$-receptor glycoprotein 130 (gp130) $(4,5)$. The former is called IL- 6 classic signaling through membrane-bound IL-6R $\alpha$ together with gp130 $(4,5)$. In contrast, the latter is called IL-6 trans-signaling by IL- 6 bound with sIL-6R $\alpha$ and IL-6/sIL$6 \mathrm{R} \alpha$ (also designated Hyper-IL-6), which is generated under pathological conditions, and signals only through gp130 as a receptor $(4,5)$. Because the IL-6/sIL-6R $\alpha$ complex is similar in molecular structure to the IL-12 family heterodimeric cytokines and has homology with the IL-12 family cytokines, IL- 6 and the IL-12 family cytokines are generally called IL-6/IL-12 family cytokines. This family of cytokines has emerged as key players in promotion and suppression of multiple immune responses under physiological and pathological situations.

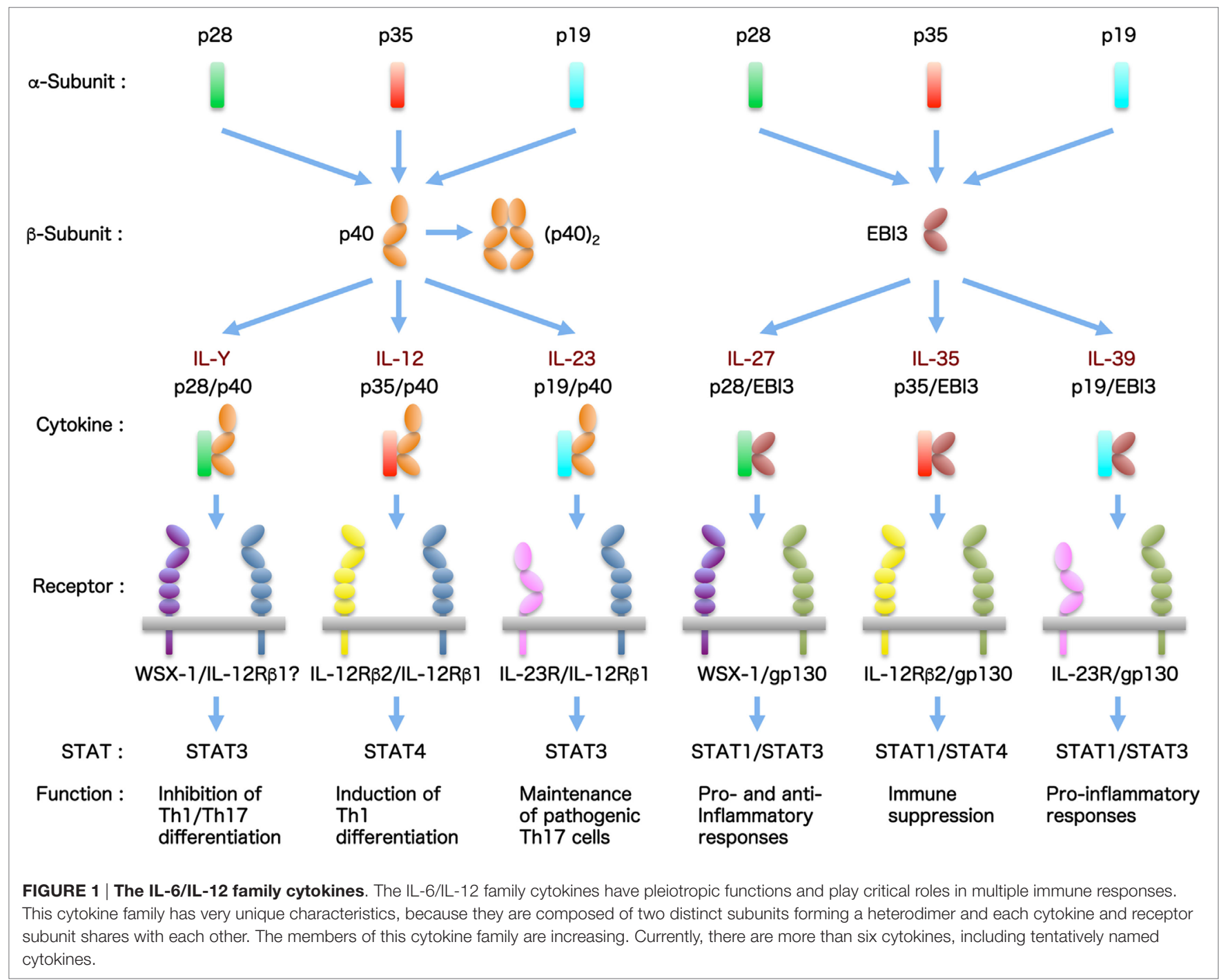


Interleukin-12 is the first member of this family and consists of two subunits, p35 connected to an intra-chain disulfide with soluble $\alpha$-receptor p40 $(6,7)$. p40 also binds to another $\beta$-subunit, 19 , by the disulfide bridge to form the second heterodimeric cytokine called IL-23 (8), whereas p40 alone forms the antagonistic or agonistic disulfide-connected homodimer p80 (9). IL-12 and IL-23 engage a heterodimeric receptor complex of IL-12R $\beta 1$ and IL-12R $\beta 2$ and of IL-12R $\beta 1$ and IL-23R, respectively, and share IL-12R $\beta 1$ for signaling (10). IL-27 consists of two subunits, p28 (IL-30) and a soluble $\alpha$-receptor EBI3, and signals via a receptor complex of WSX-1 and gp130 (11). IL-27 differs from the other IL-6/IL-12 family cytokines in that its subunits are not covalently linked. IL-35 shares the EBI3 of IL-27 and signals via four different receptor complexes: IL-12R $\beta 2 / g p 130$, IL-12R $\beta 2 / I L-12 R \beta 2$, gp130/gp130, and IL-12R $\beta 2 /$ WSX-1 $(12,13)$. IL-39, which was most recently discovered, consists of EBI3 and p19 and signals via IL-12R $\beta 1$ and IL-23R (14). p40 also binds to p28 to form p28/p40, tentatively called IL-Y, but this complex was demonstrated to be an antagonist to the signaling by IL-12 and IL-27 $(15,16)$. In addition, biological activities of monomeric forms of p28 (17), EBI3 (18), and p19 (19) and generation of soluble receptors of
WSX-1 (20) and IL-23R (21) as antagonists to IL-27 and IL-23, respectively, were reported as well.

In this review, we summarize and discuss the recent advances and expanding diversity in molecular structures and functions of the IL-6/IL-12 family cytokines (Figure 2) to enable a better understanding of them and their use as tools and targeted molecules for novel therapeutic strategies.

\section{INTERLEUKIN-27}

Interleukin-27 is a multifunctional cytokine with both proinflammatory and anti-inflammatory properties (11, 22). Although initial studies demonstrated the critical role of IL-27 in the induction of helper $\mathrm{T}$ (Th) 1 differentiation (23-27), subsequent studies revealed that IL-27 has broader stimulatory and inhibitory roles in T-cell proliferation, differentiation, cytokine production, and effector functions $(11,22)$. IL-27 prevents the development of autoimmune diseases, such as experimental autoimmune encephalomyelitis and arthritis, by inhibiting Th17 differentiation and immune responses (28). In addition, IL-27 not only promotes protective Th1 immune responses against pathogens but also suppresses them, limiting excessive inflammation

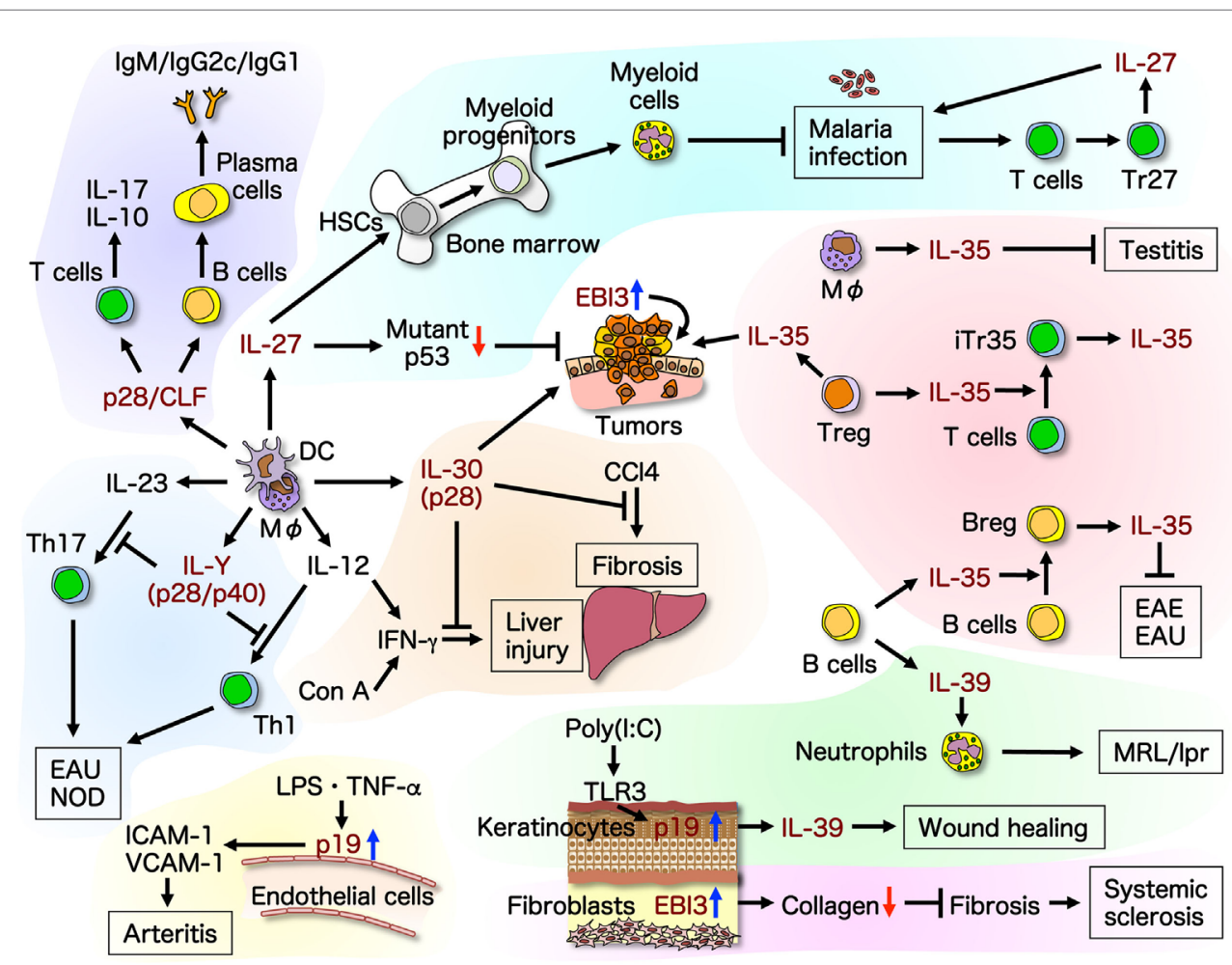

FIGURE 2 | Recent advances in molecular structures and functions of the IL-6/IL-12 family cytokines. Promiscuity within the IL-6/LL-12 family cytokines complicates the structural and functional clarification and assignment of individual cytokines. The roles of this family of cytokines are expanding, and individual cytokine subunits have been shown to have self-standing activities. This illustrates the recent advances in molecular structures and functions of the IL-6/L-12 family cytokines. Up-arrow in blue and down-arrow in red mean upregulation and downregulation of expression, respectively. Breg, regulatory B; CCI4, carbon tetrachloride; CLF, cytokine-like factor 1; Con A, concanavalin A; DC, dendritic cell; EAE, experimental autoimmune encephalomyelitis; EAU, experimental autoimmune uveitis; HSC, hematopoietic stem cell; ICAM-1, intracellular adhesion molecule-1; LPS, lipopolysaccharide; M $\Phi$, macrophage; NOD, non-obese diabetic; TLR, toll-like receptor; TNF, tumor necrosis factor; VCAM-1, vascular cell adhesion molecule-1. 
$(29,30)$ in part by IL-27-mediated induction of IL-10-producing regulatory $\mathrm{T}$ (Treg) cells $(31,32)$.

Host control of infections mainly induces Th1 responses in which interferon (IFN) $-\gamma$, IL-12, and tumor necrosis factor- $\alpha$ play critical roles in eliciting protective immunity against parasites, such as Toxoplasma and Plasmodium. However, continuing activation of effector immune cells provokes overproduction of pro-inflammatory cytokines, leading to exacerbated inflammatory reaction and lethality. Anti-inflammatory cytokines, such as IL-10 and IL-27, were demonstrated to be important for limiting the exacerbated protective Th1 responses (33-36). Moreover, malaria infection inhibits immune responses to the parasite itself, and $\mathrm{CD}^{+} \mathrm{T}$ cells from malaria-infected mice and humans have defects in the ability to produce IL-2 in response to T-cell receptor stimulation (37). Recently, a unique subpopulation of malariaspecific $\mathrm{CD}^{+} \mathrm{T}$ cells was revealed to produce IL-27 in response to T-cell receptor stimulation, subsequently inhibiting IL-2 production and clonal expansion of other T cells (38). The IL-27producing $\mathrm{CD}^{+} \mathrm{T}$ cells are forkhead box $\mathrm{P}^{-}{ }^{-} \mathrm{CD} 11 \mathrm{a}^{+} \mathrm{CD} 49 \mathrm{~d}^{+}$ malaria antigen-specific $\mathrm{CD}^{+} \mathrm{T}$ cells, and they are distinct from IFN- $\boldsymbol{\gamma}$-producing Th1 or IL-10-producing Treg cells (38). Thus, IL-27-producing regulatory $\mathrm{CD}^{+}{ }^{+} \mathrm{T}$ cells, designated $\mathrm{Tr} 27$ cells, play a critical role in the regulation of protective immune responses against malaria parasites.

During infection, cytokines play pivotal roles in both induction of protective immunity and exacerbation of inflammatory responses. Multiple mechanisms to induce protection of the host from infection have been reported. Emergency myelopoiesis, which is one of these protective responses, is inflammationinduced hematopoiesis to replenish myeloid cells in the periphery, which is critical for controlling infection with pathogens $(39,40)$. Transgenic mice expressing IL-27 previously showed enhanced myelopoiesis in the bone marrow and extramedullary hematopoiesis in the spleen (41). Hematopoietic stem cells express both IL-27R subunits, such as WSX-1 and gp130 (41). IL-27 was recently revealed to directly act on hematopoietic stem cells and promote their expansion and differentiation to myeloid progenitor cells in vitro and in vivo in synergy with stem cell factor (42). In addition, it was demonstrated that IL-27 plays an important role in the control of infection with Plasmodium berghei-attenuated variant XAT. IL-27, which is produced through IFN- $\gamma$ production during malaria infection, promotes expansion and differentiation of hematopoietic stem cells to myeloid progenitors and mobilizes them into the spleen, resulting in enhanced myelopoiesis with increased numbers of mature myeloid cells such as neutrophils (42). Thus, IL-27 is one of the limited unique cytokines directly acting on hematopoietic stem cells and promoting their expansion and differentiation into myeloid progenitor cells.

Accumulating evidence revealed the potent antitumor activities of IL-27 through multiple mechanisms, including $\mathrm{CD}^{+} \mathrm{T}$ cells, natural killer (NK) cells, antibody-dependent cell-mediated cytotoxicity, anti-angiogenesis, direct antiproliferative effect, inhibition of expression of cyclooxygenase-2 and prostaglandin $\mathrm{E}_{2}$, and suppression of epithelial-mesenchymal transition, depending on the characteristics of individual tumors (43-45). One of the most critical tumor suppressors, p53, is inherently instable and mutated in approximately 50\% of tumors, and various stressors such as DNA damage or oncogenic activation such as RAS mutations can affect the oncogenic properties of the mutant p53 (46). Because IL-27 possesses anti-inflammatory and antitumor properties, the role of endogenous IL-27 signaling in the mutant p53-mediated tumorigenesis was investigated (47). Lack of IL-27 signaling was shown to decrease the survival and double the incidence of osteosarcoma, possibly due to increased stability of the mutant p53 protein expression, indicating that IL-27 signaling negatively modulates the oncogenic properties of mutant p53 in vivo (47). In addition, lack of IL-27 signaling was demonstrated to cause spontaneous liver inflammation, fibrosis, and steatosis (48).

\section{INTERLEUKIN-35}

Interleukin-35 is preferentially secreted by forkhead box P3 ${ }^{+}$ Tregs and has suppressive activity (12). IL-35 also induces the conversion of conventional $\mathrm{T}$ cells into a suppressive IL-35producing forkhead box $\mathrm{P}^{-}$-induced Treg-cell population (termed iTr35), and this contagious spread of suppression is known as infectious tolerance (49). The receptor for IL-35 is a heterodimer of gp130 and IL-12R $\beta 2$. In addition, IL-35 has the ability to mediate signaling in the presence of only one of them as a homodimer through activation of the heterodimer or homodimer of signal transducer and activator of transcription (STAT) 1 and STAT4 (13) (Figure 3). In B cells, IL-35 was recently demonstrated to induce the expansion of a unique IL-35-producing regulatory $\mathrm{B}$ cell that conferred protection

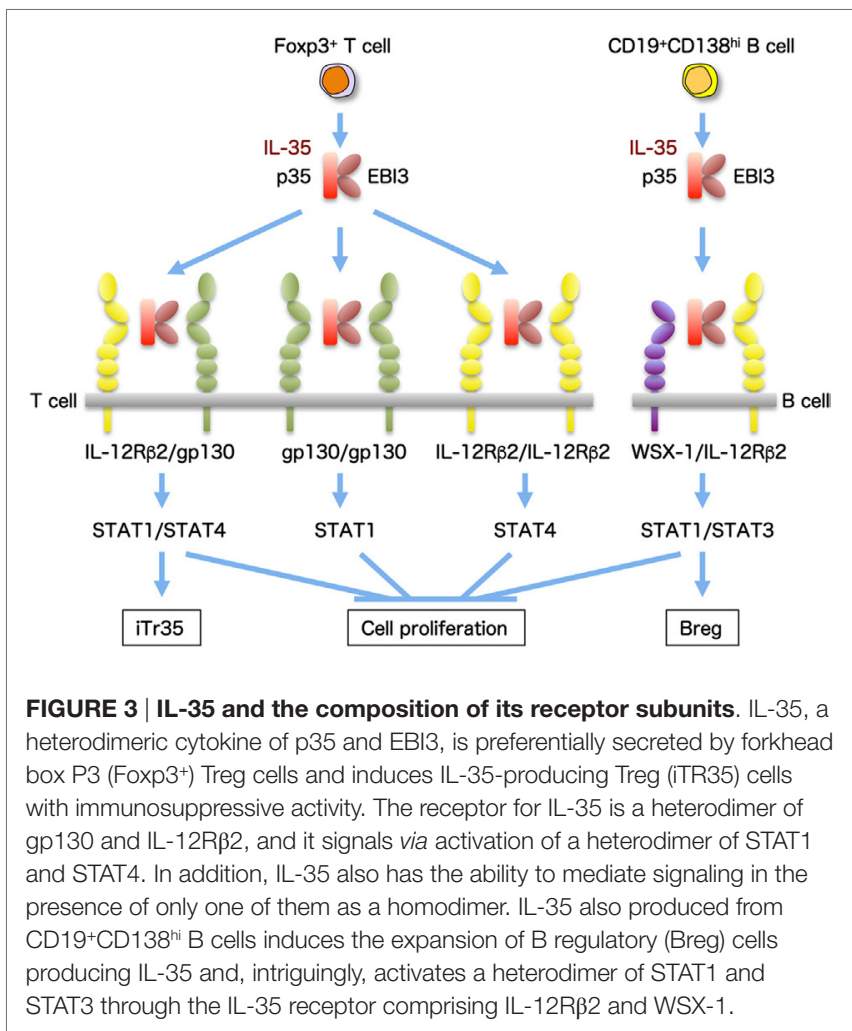


from experimental autoimmune uveitis (50). Intriguingly, IL-35 activates a heterodimer of STAT1 and STAT3 through the IL-35 receptor comprising the IL-12R $\beta 2$ and WSX-1 in B cells (50). Moreover, B cell-restricted deficiency in p35 or EBI3 exacerbated experimental autoimmune encephalomyelitis and enhanced resistance to Salmonella infection (51).

The testis has immune privilege, and the maintenance of that immune privilege status in the testes is controlled by multiple mechanisms, including the sequestration of antigens and antibodies from the immune system by the blood-testis barrier, the immunosuppressive properties of local cells, and the production of paracrine and endocrine factors $(52,53)$. Expression of EBI3 was recently demonstrated to markedly increase in the testes of 10- or 12-week-old wild-type mice as compared with levels in 2-week-old mice, whereas mRNA expression of p35 was conserved between these two groups (54). Lack of EBI3, p35, and IL-12R $\beta 2$ caused enhanced infiltration of lymphocytes into the testicular interstitium with increased IFN- $\gamma$ expression in the testes and autoantibody production against mainly acrosomal regions of spermatids (54). Immunohistochemical analyses revealed that $\mathrm{CD}_{163}{ }^{+}$resident macrophages positive for p35 and EBI3, possibly producing IL-35, were detected in the interstitium of 12 -week-old mice, but not in those of 2-week-old mice (54). These results suggest that IL-35 contributes to maintaining the testicular immune privilege.

It was previously demonstrated that there are multiple IL- $35^{+}$ cells in tumor-bearing mice and patient samples and that forced expression of IL-35 in a tumor promotes tumor progression (49, 55). Recently, the physiological impact of IL-35 on the tumor microenvironment was examined (56). Neutralization with IL-35-specific antibody or Treg cell-restricted deletion of IL-35 production limited tumor growth in multiple tumor models due to enhanced T-cell proliferation, effector function, antigenspecific responses, and long-term T-cell memory (56). Thus, IL-35 plays critical roles in preventing autoimmunity, maintaining self-tolerance, and suppressing antitumor immune responses.

\section{INTERLEUKIN-39}

Interleukin-39 is the most recently discovered cytokine. It belongs to the IL-6/IL-12 family and consists of IL-12/IL-23p19 and IL-27/IL-35EBI3, whose stable association was demonstrated in culture supernatant of activated B cells by immunoprecipitation (14). Secretion of IL-39 by activated B cells that mediate lupus-like diseases in MRL/lpr mice was demonstrated to be significantly elevated compared to other IL-12 family cytokines. In addition, adoptive transfer of activated B cells depleted of p19 or EBI3 to mice with lupus-like disease ameliorated hallmark features of systemic lupus erythematosus, including reduction of splenomegaly, pathogenic B cells, and proteinuria (14). IL-39 was also shown to induce differentiation and/or expansion of neutrophils, whose ability is critically important for induction of pathogenic features of the autoimmune disease (57). Moreover, IL-39-induced neutrophils had positive feedback on IL-39 expression in activated B cells by secreting B-cell activation factor (57). Thus, IL-39 secreted by activated B cells may be an important pro-inflammatory cytokine and a potential therapeutic target for the treatment of autoimmune diseases such as systemic lupus erythematosus.

A similar possible association between p19 and EBI3 was suggested in damaged keratinocytes (58). In keratinocytes, toll-like receptor 3 has an important role in detecting damageassociated molecular patterns released from damaged cells and in initiating cell repair processes (59). Toll-like receptor 3-mediated activation of keratinocytes was demonstrated to drive IFN regulatory factor 6-dependent p19 expression and p19/EBI3 heterodimer formation, possibly contributing to wound healing by damping inflammatory responses (58). Further studies are necessary to clarify whether IL-39 is a pro-inflammatory cytokine or an anti-inflammatory cytokine, or both, in keratinocytes.

\section{IL-Y (p28/p40)}

The $\alpha$-subunit of IL-27, p28, which is referred to as IL-30, was initially reported to inhibit gp130 signaling by directly binding to gp130, resulting in inhibition of Th17 differentiation as an antagonist (60). However, IL-30 was demonstrated to function as an agonistic cytokine via the gp130 signaling pathway similar to IL-6, either by itself or associated with other molecules, such as IL-12p40 $(15,16)$, cytokine-like factor 1 (CLF) (61), IL-6R $\alpha$ (62), forming p28/IL-6R $\alpha$ classic signaling, p28/sIL-6R $\alpha$ transsignaling, and p28 signaling (Figure 4).

After identification of IL-35 in 2007 (12), additional potential pairings were examined; one of these candidates was the IL-27p28/IL-12p40 heterodimer (15). Initial studies using Western blot followed by immunoprecipitation reaction revealed that a stable association between p28 and p40 was formed possibly via disulfide bond (15). The therapeutic effect of p28/ p40 was then examined using a mouse model of experimental autoimmune uveitis, which is caused by Th1 and Th17 cells. Injection of p28/p40 protein suppressed experimental autoimmune uveitis by inhibiting the differentiation and inflammatory responses of Th1 and Th17 cells (15). These suppressive effects seemed to be ascribed to antagonizing the activation of STAT1 and STAT3 pathways induced by IL-27 and IL-6, both of which signal through the gp130 receptor (15). Moreover, recent studies using adenovirus vector expressing p28/p40 (IL-Y) suggested that treatment of prediabetic non-obese diabetic mice prevented the onset of hyperglycemia with reduced expression of inflammatory mediators such as IFN- $\gamma$ (16). Interestingly, in this study, p28/p40 was demonstrated to be able to significantly stimulate a unique cytokine and chemokine expression profile as well as to activate STAT3, in part, through a pathway involving WSX-1 (16). These results implicate that the p28/p40 might be a bioactive immunosuppressive cytokine and not a mere competitive inhibitor against IL-27, IL-12, or IL-23.

\section{p28/CLF}

Independently of EBI3, p28 also forms a complex with CLF, and the resultant $\mathrm{p} 28 / \mathrm{CLF}$ complex is produced by activated dendritic cells (61). CLF is a soluble cytokine receptor that forms a complex with cardiotrophin-like cytokines, which have 


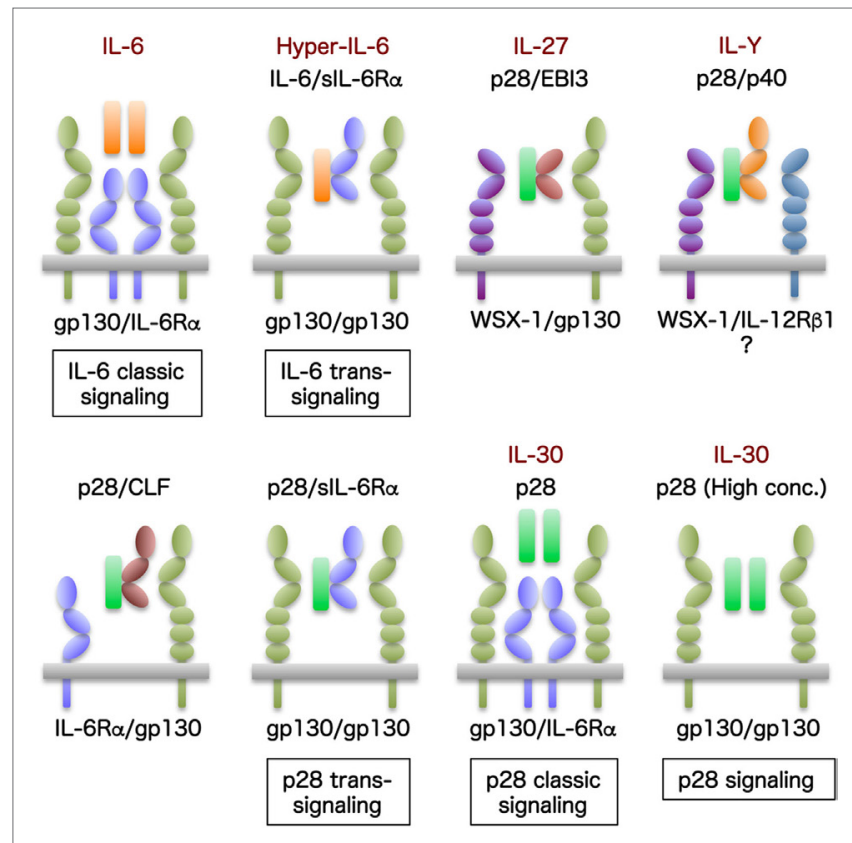

FIGURE 4 | IL-30 and its related molecules. IL-6 is unique in signaling through not only membrane-bound IL-6R $\alpha$ but also sIL-6R $\alpha$. The former is called IL-6 classic signaling through membrane-bound IL-6R $\alpha$ together with gp130, and the latter is called IL-6 trans-signaling by IL-6 bound with sIL-6R $\alpha$ and IL-6/sIL-6R $\alpha$ (also designated Hyper-IL-6) through only gp130. The $\alpha$ subunit of IL-27, p28, which is referred to as IL-30, functions as an agonistic cytokine via the gp130 signaling pathway similar to IL-6, either by itself or associating with other molecules such as IL-12p40, CLF, IL-6R $\alpha$, forming p28/IL-6R $\alpha$ classic signaling, p28/sIL-6R $\alpha$ trans-signaling, and p28 signaling. Moreover, p28 can activate signal transduction via gp130, even in the absence of IL-6R $\alpha$ or EBI3, albeit at higher concentrations.

neurotrophic and immunomodulatory activities, for efficient secretion (63). p28/CLF was demonstrated to activate NK cells with IL-12-induced and IL-2-induced IFN- $\gamma$ production and to induce activation of STAT1 and STAT3, which requires gp130 and IL-6R $\alpha$, but not WSX-1, as receptor subunits for signaling in $\mathrm{CD}^{+}$and $\mathrm{CD}^{+} \mathrm{T}$ cells (61). It also promoted IL-17 and IL-10 secretion from $\mathrm{CD}^{+} \mathrm{T}$ cells and inhibited their proliferation. In $\mathrm{B}$ cells, p28/CLF enhanced their proliferation and induced differentiation into plasma cells producing IgM, IgG2c, and IgG1 (64). In the factor-dependent B-cell line, Ba/F3 cells expressing gp130 and IL-6R $\alpha$, p28/CLF induced activation of STAT1 and STAT3 and their proliferation (61). Similar effects were also observed with p28 alone, indicating that p28 alone is biologically active in cells expressing gp130 and IL-6R $\alpha$, such as hepatocytes like IL-6 $(61,62)$. This is because CLF is assumed to be necessary only for secretion of p28, but not for p28/IL-6R $\alpha$-mediated signaling $(61,62)$.

\section{p28/sIL-6R $\alpha$ (p28 TRANS-SIGNALING)}

Because IL-6R $\alpha$ is only expressed in hepatocytes and subtypes of lymphocytes, but gp130 is almost ubiquitously expressed, IL-6 can signal via not only the membrane-bound IL-6R $\alpha$ and gp130 (IL-6 classic signaling) but also the sIL-6R $\alpha$ through only gp130 (IL-6 trans-signaling); serum levels of sIL-6R $\alpha$ are increased under inflammatory conditions and the spectrum of IL-6-responsive cells expands to almost all types of cells throughout the body $(2,5)$. Similarly, p28 can also induce signaling via membrane-bound IL-6R $\alpha$; therefore, p28 was speculated to induce trans-signaling via sIL-6R $\alpha$ (61). Indeed, p28/sIL-6R $\alpha$ fusion protein similar to IL-6/sIL-6R $\alpha$ (Hyper-IL-6) was shown to induce phosphorylation of STAT3 and proliferation of $\mathrm{Ba} / \mathrm{F} 3$ cells expressing gp130, which are inhibited by soluble gp130 (62). Although the findings of $\mathrm{p} 28 / \mathrm{sIL}-6 \mathrm{R} \alpha$ trans-signaling expand the spectrum of its responsive cells to virtually all cells in the body, such as IL-6, physiological roles of the p28/sIL-6R $\alpha$ transsignaling remain to be clarified.

\section{IL-30 (p28) (p28 CLASSIC SIGNALING)}

Although IL-27 signals via a heterodimer of gp130 and WSX-1, p28/IL-6R $\alpha$ was demonstrated to specifically recruit two gp130 receptors for signal transduction (62). The binding of p28 to a gp130/WSX-1 heterodimer or a gp130 homodimer was revealed to be highly selective and controlled by a novel molecular switch induced by EBI3 (as IL-27) or IL-6R $\alpha$ (as p28 classic signaling), respectively (62). Moreover, because p28 has an intrinsic affinity for gp130 (60), p28 was able to activate signal transduction via gp130, even in the absence of IL-6R $\alpha$ or EBI3, albeit at higher concentrations (62).

Recent evidence suggested that IL-30 also acts as an IL-27independent self-standing cytokine with its own functions in addition to associating with EBI3 to form IL-27. These functions include a hepatoprotective role against liver injury and liver fibrosis induced by acute and chronic inflammation with IL-12, IFN- $\gamma$, concanavalin A, and carbon tetrachloride (65-67). This is consistent with the fact that IL-6R $\alpha$ is mainly expressed on hepatocytes $(4,5,62)$. Injection of the IL-30 expression vector was demonstrated to inhibit IL-12-induced and concanavalin A-induced liver injury due to suppression of IFN- $\gamma$ production (65). Consistent with this, dendritic cell-specific p28 conditional knockout mice exacerbated concanavalin A-induced liver injury with higher production of IFN- $\gamma$ from $\mathrm{CD} 4^{+} \mathrm{T}$ cells, but not NKT cells (66). In addition, injection of the IL-30 expression vector attenuated liver fibrosis by recruiting NKT cells into the liver to remove activated hepatic stellate cells through an NKG2D-Rae1 interaction (67).

Moreover, IL-30, but not EBI3, was demonstrated to be expressed in prostate cancer lesions and tumor-draining lymph nodes, such as $\mathrm{CD}^{+} 8^{+}$macrophages, $\mathrm{CD} 33^{+} / \mathrm{CD} 11 \mathrm{~b}^{+}$myeloid cells, and CD14 monocytes, and its expression correlated with advanced disease grade and stage (68). In addition, IL-30 stimulated proliferation of human prostate cancer cells, which express both IL-6R $\alpha$ and gp130, and downregulated the expression of chemokines, such as CCL16, tumor necrosis factor superfamily member 14 , and chemokine-like factor, which recruit immune cells into the tumor (68). IL-30 also upregulated the tumor suppressor and androgen co-receptor CKLF-like MARVEL transmembrane domain containing-3 and multifunctional receptor chemokine-like receptor-1 (68). Thus, IL-30 may be an important cytokine shaping the tumor and lymph node microenvironment. 


\section{EPSTEIN-BARR VIRUS-INDUCED PROTEIN 3}

Epstein-Barr virus-induced protein 3 was first identified in B lymphocytes as a gene whose expression is induced by EpsteinBarr virus infection and is readily secreted by itself (69). Recently, evidence revealed that EBI3 may also function as a self-standing molecule. It was demonstrated that high expression of EBI3 in lung cancer patients is associated with a poor prognosis and that serum levels of EBI3 in lung cancer patients are significantly higher than those in healthy volunteers (70). Furthermore, reduction of EBI3 expression by siRNA suppressed cancer cell proliferation and induction of exogenous EBI3 expression conferred growthpromoting activity (70).

Systemic sclerosis is a connective tissue disorder characterized by fibrosis of the skin and caused by the activation of fibroblast and excessive deposition of the extracellular matrix, mainly type I collagen (71). Expression of EBI3, but not IL-35, was recently demonstrated to be decreased in the keratinocytes of the epidermis and Treg cells of the dermis in systemic sclerosis skin compared with normal skin, whereas injection of EBI3 alone into the skin improved mice skin fibrosis and addition of EBI3 alone in cultured dermal fibroblasts decreased type I collagen expression (18). Although the possibility that EBI3 may form a complex with endogenous p 35 or other molecule cannot be excluded, EBI3 itself may directly affect collagen expression.

CsEBI3, a fish EBI3 homolog, was recently identified from tongue sole (Cynoglossus semilaevis) and demonstrated immunostimulatory properties depending on the conserved fibronectin type III domain as a self-standing cytokine (72). Bacterial infection of peripheral blood leukocytes enhanced CsEBI3 expression and caused extracellular secretion of $\mathrm{CsEBI} 3$, and purified recombinant CsEBI3 stimulated the respiratory burst activity of peripheral blood leukocytes and upregulated the expression of IL-1 $\beta$, IL-8, myeloid differentiation primary response gene 88 , IFN-induced gene 15, CD28, and chemokines (72). Thus, the EBI3 homolog alone may play a critical role in the antimicrobial host defense in fish.

\section{p19}

Self-standing activity of intracellular IL-23p19 was recently reported to play important roles as an endogenous activator of endothelial inflammation, promoting leukocyte adhesion to endothelial cells and transendothelial migration (19). Inflammatory mediators play important roles in the pathogenesis of vascular lesions that characterize different types of vasculitis, including giant-cell arteritis (73). It was recently demonstrated that p19 expression is enhanced by lipopolysaccharides or tumor necrosis factor- $\alpha$ in the absence of p40 in endothelial cells and that intracellular expression of p19 increases their cell surface expression of intracellular adhesion molecule-1 and vascular cell adhesion molecule-1, which enhance the attachment of leukocytes and increase their transendothelial migration (19). Intriguingly, the intracellular expression of p19 associated with cytokine receptor subunit gp130 and stimulated gp130-dependent activation of STAT3 signaling. In addition, endothelial p19 expression also associated with gp130 in the adventitial capillaries of inflamed temporal arteries of patients with giant-cell arteritis that do not contain p40 (19). However, how p19-gp130 interaction elicits STAT3 activation remains to be clarified.

\section{CONCLUSION AND FUTURE PERSPECTIVES}

Most of the cytokines identified to date have mainly proinflammatory property, and there are only a few cytokines with anti-inflammatory properties, including IL-10, transforming growth factor- $\beta$, IL-27, and IL-35. Among them, IL-35 may be the only cytokine that possesses anti-inflammatory property alone. IL-10 is best known as an anti-inflammatory cytokine, but its immunosuppressive function is mainly limited to antigenpresenting cells (74). For $\mathrm{CD}^{+} \mathrm{T}$ cells and $\mathrm{B}$ cells, IL-10 has pro-inflammatory functions and promotes proliferation and antibody production (74). This "double-edged sword" property significantly hinders clinical application of IL-10 because such pro-inflammatory functions might lead to adverse effects. So far, only the anti-inflammatory property is known for IL-35; therefore, this cytokine could be one of the most promising candidates for clinical application against allergic and autoimmune diseases. However, among the IL-6/IL-12 family cytokines, IL-35 is considered to differ from the other cytokines (75). Although bacterially produced and purified recombinant proteins of EBI3 and p35 were correctly folded and biologically active in combination with p 28 and p40, respectively, no biologically active IL-35 was reported to be formed when the p35 and EBI3 were combined (75). There are currently no reasons to explain this, but IL-35 might need additional, yet unidentified, molecules for efficient secretion and exertion of biological activity (75). One of the best criteria to prove the relationship between a bioactive cytokine and its receptor should be to examine whether the recombinant cytokine can proliferate a factor-dependent cell line such as $\mathrm{Ba} / \mathrm{F} 3$ cells expressing its receptor subunits. $\mathrm{Ba} / \mathrm{F} 3$ is an IL-3-dependent mouse pro-B cell line used very commonly for assessing the potency of biologically active signaling molecules, including cytokines and kinases. In addition to IL-35, IL-Y, IL-39, and new cytokines to be identified in the near future need such studies to confirm the biological relationship.

Thus, the diversity in molecular structures and functions of the IL-6/IL-12 family cytokines is still expanding in various physiological and pathological situations. Although the Human Genome Project was declared complete in 2003, and approximately 20,000 genes were identified, this number is much less than expected $(76,77)$. This gap is considered to be caused by the differences in subunit structure and alternative splicing. Growing diversity in the molecular structures and functions of the IL-6/IL-12 family cytokines should significantly contribute to filling the gap. Currently, the newest cytokine is IL-39, but some cytokine family members might be renamed because of new interleukins that might be found in the near future. 


\section{AUTHOR CONTRIBUTIONS}

$\mathrm{HH}$ and TY organized and wrote the manuscript. IM and MX designed and drew the figures. YC and MO commented on the manuscript.

\section{REFERENCES}

1. Vignali DA, Kuchroo VK. IL-12 family cytokines: immunological playmakers. Nat Immunol (2012) 13(8):722-8. doi:10.1038/ni.2366

2. Garbers C, Hermanns HM, Schaper F, Muller-Newen G, Grotzinger J, Rose-John S, et al. Plasticity and cross-talk of interleukin 6-type cytokines. Cytokine Growth Factor Rev (2012) 23(3):85-97. doi:10.1016/j.cytogfr.2012. 04.001

3. Belladonna ML, Grohmann U. Bioengineering heterodimeric cytokines: turning promiscuous proteins into therapeutic agents. Biotechnol Genet Eng $\operatorname{Rev}(2013)$ 29:149-74. doi:10.1080/02648725.2013.801228

4. Schaper F, Rose-John S. Interleukin-6: biology, signaling and strategies of blockade. Cytokine Growth Factor Rev (2015) 26(5):475-87. doi:10.1016/j. cytogfr.2015.07.004

5. Scheller J, Chalaris A, Schmidt-Arras D, Rose-John S. The pro- and antiinflammatory properties of the cytokine interleukin-6. Biochim Biophys Acta (2011) 1813(5):878-88. doi:10.1016/j.bbamcr.2011.01.034

6. Gately MK, Renzetti LM, Magram J, Stern AS, Adorini L, Gubler U, et al. The interleukin-12/interleukin-12-receptor system: role in normal and pathologic immune responses. Annu Rev Immunol (1998) 16:495-521. doi:10.1146/ annurev.immunol.16.1.495

7. Trinchieri G. Interleukin-12 and the regulation of innate resistance and adaptive immunity. Nat Rev Immunol (2003) 3(2):133-46. doi:10.1038/ nri1001

8. Oppmann B, Lesley R, Blom B, Timans JC, Xu Y, Hunte B, et al. Novel p19 protein engages IL-12p40 to form a cytokine, IL-23, with biological activities similar as well as distinct from IL-12. Immunity (2000) 13(5):715-25. doi:10.1016/S1074-7613(00)00070-4

9. Cooper AM, Khader SA. IL-12p40: an inherently agonistic cytokine. Trends Immunol (2007) 28(1):33-8. doi:10.1016/j.it.2006.11.002

10. Teng MW, Bowman EP, McElwee JJ, Smyth MJ, Casanova JL, Cooper AM, et al. IL-12 and IL-23 cytokines: from discovery to targeted therapies for immune-mediated inflammatory diseases. Nat Med (2015) 21(7):719-29. doi:10.1038/nm.3895

11. Yoshida H, Hunter CA. The immunobiology of interleukin-27. Annu Rev Immunol (2015) 33:417-43. doi:10.1146/annurev-immunol-032414-112134

12. Collison LW, Workman CJ, Kuo TT, Boyd K, Wang Y, Vignali KM, et al. The inhibitory cytokine IL-35 contributes to regulatory T-cell function. Nature (2007) 450(7169):566-9. doi:10.1038/nature06306

13. Collison LW, Delgoffe GM, Guy CS, Vignali KM, Chaturvedi V, Fairweather D, et al. The composition and signaling of the IL-35 receptor are unconventional. Nat Immunol (2012) 13(3):290-9. doi:10.1038/ni.2227

14. Wang X, Wei Y, Xiao H, Liu X, Zhang Y, Han G, et al. A novel IL-23p19/Ebi3 (IL-39) cytokine mediates inflammation in lupus-like mice. Eur J Immunol (2016) 46(6):1343-50. doi:10.1002/eji.201546095

15. Wang RX, Yu CR, Mahdi RM, Egwuagu CE. Novel IL27p28/IL12p40 cytokine suppressed experimental autoimmune uveitis by inhibiting autoreactive Th1/ Th17 cells and promoting expansion of regulatory T cells. J Biol Chem (2012) 287(43):36012-21. doi:10.1074/jbc.M112.390625

16. Flores RR, Kim E, Zhou L, Yang C, Zhao J, Gambotto A, et al. IL-Y, a synthetic member of the IL-12 cytokine family, suppresses the development of type 1 diabetes in NOD mice. Eur J Immunol (2015) 45(11):3114-25. doi:10.1002/ eji.201445403

17. Yan J, Mitra A, Hu J, Cutrera JJ, Xia X, Doetschman T, et al. Interleukin-30 (IL27p28) alleviates experimental sepsis by modulating cytokine profile in NKT cells. J Hepatol (2016) 64(5):1128-36. doi:10.1016/j.jhep.2015. 12.020

18. Kudo H, Wang Z, Jinnin M, Nakayama W, Inoue K, Honda N, et al. EBI3 downregulation contributes to type I collagen overexpression in scleroderma skin. J Immunol (2015) 195(8):3565-73. doi:10.4049/jimmunol.1402362

\section{FUNDING}

This study was supported in part by grants from the Ministry of Education, Culture, Sports, Science, and Technology, Japan.

19. Espigol-Frigole G, Planas-Rigol E, Ohnuki H, Salvucci O, Kwak H, Ravichandran S, et al. Identification of IL-23p19 as an endothelial proinflammatory peptide that promotes gp130-STAT3 signaling. Sci Signal (2016) 9(419):ra28. doi:10.1126/scisignal.aad2357

20. Dietrich C, Candon S, Ruemmele FM, Devergne O. A soluble form of IL-27Ralpha is a natural IL-27 antagonist. J Immunol (2014) 192(11):5382-9. doi:10.4049/jimmunol.1303435

21. Franke M, Schroder J, Monhasery N, Ackfeld T, Hummel TM, Rabe B, et al. Human and murine interleukin 23 receptors are novel substrates for a disintegrin and metalloproteases ADAM10 and ADAM17. J Biol Chem (2016) 291(20):10551-61. doi:10.1074/jbc.M115.710541

22. Hunter CA, Kastelein R. Interleukin-27: balancing protective and pathological immunity. Immunity (2012) 37(6):960-9. doi:10.1016/j.immuni.2012. 11.003

23. Pflanz S, Timans JC, Cheung J, Rosales R, Kanzler H, Gilbert J, et al. IL-27, a heterodimeric cytokine composed of EBI3 and p28 protein, induces proliferation of naive CD4+ T cells. Immunity (2002) 16(6):779-90. doi:10.1016/ S1074-7613(02)00324-2

24. Pflanz S, Hibbert L, Mattson J, Rosales R, Vaisberg E, Bazan JF, et al. WSX-1 and glycoprotein 130 constitute a signal-transducing receptor for IL-27. J Immunol (2004) 172(4):2225-31. doi:10.4049/jimmunol.172.4.2225

25. Owaki T, Asakawa M, Morishima N, Hata K, Fukai F, Matsui M, et al. A role for IL-27 in early regulation of Th1 differentiation. J Immunol (2005) 175(4):2191-200. doi:10.4049/jimmunol.175.4.2191

26. Owaki T, Asakawa M, Fukai F, Mizuguchi J, Yoshimoto T. IL-27 induces Th1 differentiation via p38 MAPK/T-bet- and intercellular adhesion molecule-1/ LFA-1/ERK1/2-dependent pathways. J Immunol (2006) 177(11):7579-87. doi:10.4049/jimmunol.177.11.7579

27. Owaki T, Asakawa M, Morishima N, Mizoguchi I, Fukai F, Takeda K, et al. STAT3 is indispensable to IL-27-mediated cell proliferation but not to IL-27induced Th1 differentiation and suppression of proinflammatory cytokine production. J Immunol (2008) 180(5):2903-11. doi:10.4049/jimmunol.180. 5.2903

28. Awasthi A, Carrier Y, Peron JP, Bettelli E, Kamanaka M, Flavell RA, et al. A dominant function for interleukin 27 in generating interleukin 10-producing anti-inflammatory T cells. Nat Immunol (2007) 8(12):1380-9. doi:10.1038/ni1541

29. Villarino A, Hibbert L, Lieberman L, Wilson E, Mak T, Yoshida H, et al. The IL-27R (WSX-1) is required to suppress $\mathrm{T}$ cell hyperactivity during infection. Immunity (2003) 19(5):645-55. doi:10.1016/S1074-7613(03) 00300-5

30. Hamano S, Himeno K, Miyazaki Y, Ishii K, Yamanaka A, Takeda A, et al. WSX-1 is required for resistance to Trypanosoma cruzi infection by regulation of proinflammatory cytokine production. Immunity (2003) 19(5):657-67. doi:10.1016/S1074-7613(03)00298-X

31. Pot C, Apetoh L, Awasthi A, Kuchroo VK. Induction of regulatory Tr1 cells and inhibition of T(H)17 cells by IL-27. Semin Immunol (2011) 23(6):438-45. doi:10.1016/j.smim.2011.08.003

32. Pot C, Apetoh L, Kuchroo VK. Type 1 regulatory T cells (Tr1) in autoimmunity. Semin Immunol (2011) 23(3):202-8. doi:10.1016/j.smim.2011. 07.005

33. Gazzinelli RT, Wysocka M, Hieny S, Scharton-Kersten T, Cheever A, Kuhn $\mathrm{R}$, et al. In the absence of endogenous IL-10, mice acutely infected with Toxoplasma gondii succumb to a lethal immune response dependent on CD4+ $\mathrm{T}$ cells and accompanied by overproduction of IL-12, IFN-gamma and TNFalpha. J Immunol (1996) 157(2):798-805.

34. Findlay EG, Greig R, Stumhofer JS, Hafalla JC, de Souza JB, Saris CJ, et al. Essential role for IL-27 receptor signaling in prevention of Th1-mediated immunopathology during malaria infection. J Immunol (2010) 185(4): 2482-92. doi:10.4049/jimmunol.0904019 
35. Freitas do Rosario AP, Lamb T, Spence P, Stephens R, Lang A, Roers A, et al. IL-27 promotes IL-10 production by effector Th1 CD4+ T cells: a critical mechanism for protection from severe immunopathology during malaria infection. J Immunol (2012) 188(3):1178-90. doi:10.4049/jimmunol.1102755

36. Villegas-Mendez A, de Souza JB, Lavelle SW, Gwyer Findlay E, Shaw TN, van Rooijen N, et al. IL-27 receptor signalling restricts the formation of pathogenic, terminally differentiated Thl cells during malaria infection by repressing IL-12 dependent signals. PLoS Pathog (2013) 9(4):e1003293. doi:10.1371/journal.ppat.1003293

37. Ho M, Webster HK, Green B, Looareesuwan S, Kongchareon S, White NJ. Defective production of and response to IL-2 in acute human falciparum malaria. J Immunol (1988) 141(8):2755-9.

38. Kimura D, Miyakoda M, Kimura K, Honma K, Hara H, Yoshida H, et al. Interleukin-27-producing CD4(+) T cells regulate protective immunity during malaria parasite infection. Immunity (2016) 44(3):672-82. doi:10.1016/j. immuni.2016.02.011

39. Takizawa H, Boettcher S, Manz MG. Demand-adapted regulation of early hematopoiesis in infection and inflammation. Blood (2012) 119(13): 2991-3002. doi:10.1182/blood-2011-12-380113

40. Manz MG, Boettcher S. Emergency granulopoiesis. Nat Rev Immunol (2014) 14(5):302-14. doi:10.1038/nri3660

41. Seita J, Asakawa M, Ooehara J, Takayanagi S, Morita Y, Watanabe N, et al. Interleukin-27 directly induces differentiation in hematopoietic stem cells. Blood (2008) 111(4):1903-12. doi:10.1182/blood-2007-06-093328

42. Furusawa J, Mizoguchi I, Chiba Y, Hisada M, Kobayashi F, Yoshida H, et al. Promotion of expansion and differentiation of hematopoietic stem cells by interleukin-27 into myeloid progenitors to control infection in emergency myelopoiesis. PLoS Pathog (2016) 12(3):e1005507. doi:10.1371/journal.ppat. 1005507

43. Hisada M, Kamiya S, Fujita K, Belladonna ML, Aoki T, Koyanagi Y, et al. Potent antitumor activity of interleukin-27. Cancer Res (2004) 64(3):1152-6. doi:10.1158/0008-5472.CAN-03-2084

44. Yoshimoto T, Chiba Y, Furusawa JI, Xu M, Tsunoda R, Higuchi K, et al. Potential clinical application of interleukin-27 as an antitumor agent. Cancer Sci (2015) 106(9):1103-10. doi:10.1111/cas.12731

45. Mizoguchi I, Chiba Y, Furusawa JI, Xu M, Tsunoda R, Higuchi K, et al. Therapeutic potential of interleukin-27 against cancers in preclinical mouse models. Oncoimmunology (2015) 4(10):e1042200. doi:10.1080/2162402X. 2015.1042200

46. Suh YA, Post SM, Elizondo-Fraire AC, Maccio DR, Jackson JG, El-Naggar AK, et al. Multiple stress signals activate mutant p53 in vivo. Cancer Res (2011) 71(23):7168-75. doi:10.1158/0008-5472.CAN-11-0459

47. Dibra D, Mitra A, Newman M, Xia X, Cutrera JJ, Gagea M, et al. Lack of immunomodulatory interleukin-27 enhances oncogenic properties of mutant p53 in vivo. Clin Cancer Res (2016) 22(15):3876-83. doi:10.1158/1078-0432. CCR-15-2052

48. Dibra D, Xia X, Mitra A, Cutrera JJ, Lozano G, Li S. Mutant p53 in concert with an interleukin-27 receptor alpha deficiency causes spontaneous liver inflammation, fibrosis, and steatosis in mice. Hepatology (2016) 63(3):1000-12. doi:10.1002/hep.28379

49. Collison LW, Chaturvedi V, Henderson AL, Giacomin PR, Guy C, Bankoti J, et al. IL-35-mediated induction of a potent regulatory T cell population. Nat Immunol (2010) 11(12):1093-101. doi:10.1038/ni.1952

50. Wang RX, Yu CR, Dambuza IM, Mahdi RM, Dolinska MB, Sergeev YV, et al. Interleukin-35 induces regulatory B cells that suppress autoimmune disease. Nat Med (2014) 20(6):633-41. doi:10.1038/nm.3554

51. Shen P, Roch T, Lampropoulou V, O'Connor RA, Stervbo U, Hilgenberg E, et al. IL-35-producing B cells are critical regulators of immunity during autoimmune and infectious diseases. Nature (2014) 507(7492):366-70. doi:10.1038/nature 12979

52. Fijak M, Meinhardt A. The testis in immune privilege. Immunol Rev (2006) 213:66-81. doi:10.1111/j.1600-065X.2006.00438.x

53. Li N, Wang T, Han D. Structural, cellular and molecular aspects of immune privilege in the testis. Front Immunol (2012) 3:152. doi:10.3389/ fimmu.2012.00152

54. Terayama H, Yoshimoto T, Hirai S, Naito M, Qu N, Hatayama N, et al. Contribution of IL-12/IL-35 common subunit p35 to maintaining the testicular immune privilege. PLoS One (2014) 9(4):e96120. doi:10.1371/journal. pone.0096120
55. Wang Z, Liu JQ, Liu Z, Shen R, Zhang G, Xu J, et al. Tumor-derived IL-35 promotes tumor growth by enhancing myeloid cell accumulation and angiogenesis. J Immunol (2013) 190(5):2415-23. doi:10.4049/jimmunol. 1202535

56. Turnis ME, Sawant DV, Szymczak-Workman AL, Andrews LP, Delgoffe GM, Yano H, et al. Interleukin-35 limits anti-tumor immunity. Immunity (2016) 44(2):316-29. doi:10.1016/j.immuni.2016.01.013

57. Wang X, Liu X, Zhang Y, Wang Z, Zhu G, Han G, et al. IL-39 (IL-23p19/Ebi3) induces differentiation/expansion of neutrophils in lupus-prone mice. Clin Exp Immunol (2016) 186(2):144-56. doi:10.1111/cei.12840

58. Ramnath D, Tunny K, Hohenhaus DM, Pitts CM, Bergot AS, Hogarth PM, et al. TLR3 drives IRF6-dependent IL-23p19 expression and p19/EBI3 heterodimer formation in keratinocytes. Immunol Cell Biol (2015) 93(9):771-9. doi:10.1038/icb.2015.77

59. Lin Q, Fang D, Fang J, Ren X, Yang X, Wen F, et al. Impaired wound healing with defective expression of chemokines and recruitment of myeloid cells in TLR3-deficient mice. J Immunol (2011) 186(6):3710-7. doi:10.4049/ jimmunol.1003007

60. Stumhofer JS, Tait ED, Quinn WJ III, Hosken N, Spudy B, Goenka R, et al. A role for IL-27p28 as an antagonist of gp130-mediated signaling. Nat Immunol (2010) 11(12):1119-26. doi:10.1038/ni.1957

61. Crabe S, Guay-Giroux A, Tormo AJ, Duluc D, Lissilaa R, Guilhot F, et al. The IL-27 p28 subunit binds cytokine-like factor 1 to form a cytokine regulating $\mathrm{NK}$ and $\mathrm{T}$ cell activities requiring IL-6R for signaling. J Immunol (2009) 183(12):7692-702. doi:10.4049/jimmunol.0901464

62. Garbers C, Spudy B, Aparicio-Siegmund S, Waetzig GH, Sommer J, Holscher C, et al. An interleukin-6 receptor-dependent molecular switch mediates signal transduction of the IL-27 cytokine subunit p28 (IL-30) via a gp130 receptor homodimer. J Biol Chem (2013) 288(6):4346-54. doi:10.1074/jbc. M112.432955

63. Tormo AJ, Letellier MC, Lissilaa R, Batraville LA, Sharma M, Ferlin W, et al. The cytokines cardiotrophin-like cytokine/cytokine-like factor-1 (CLC/CLF) and ciliary neurotrophic factor (CNTF) differ in their receptor specificities. Cytokine (2012) 60(3):653-60. doi:10.1016/j.cyto.2012.08.014

64. Tormo AJ, Meliani Y, Beaupre LA, Sharma M, Fritz JH, Elson G, et al. The composite cytokine p28/cytokine-like factor 1 sustains B cell proliferation and promotes plasma cell differentiation. J Immunol (2013) 191(4):1657-65. doi:10.4049/jimmunol.1201595

65. Dibra D, Cutrera J, Xia X, Kallakury B, Mishra L, Li S. Interleukin-30: a novel antiinflammatory cytokine candidate for prevention and treatment of inflammatory cytokine-induced liver injury. Hepatology (2012) 55(4):1204-14. doi:10.1002/hep. 24814

66. Zhang S, Liang R, Luo W, Liu C, Wu X, Gao Y, et al. High susceptibility to liver injury in IL-27 p28 conditional knockout mice involves intrinsic interferon-gamma dysregulation of CD4+ T cells. Hepatology (2013) 57(4): 1620-31. doi:10.1002/hep.26166

67. Mitra A, Satelli A, Yan J, Xueqing X, Gagea M, Hunter CA, et al. IL-30 (IL27p28) attenuates liver fibrosis through inducing NKG2D-rae1 interaction between NKT and activated hepatic stellate cells in mice. Hepatology (2014) 60(6):2027-39. doi:10.1002/hep.27392

68. Di Meo S, Airoldi I, Sorrentino C, Zorzoli A, Esposito S, Di Carlo E. Interleukin-30 expression in prostate cancer and its draining lymph nodes correlates with advanced grade and stage. Clin Cancer Res (2014) 20(3):585-94. doi:10.1158/1078-0432.CCR-13-2240

69. Devergne O, Hummel M, Koeppen H, Le Beau MM, Nathanson EC, Kieff E, et al. A novel interleukin-12 p40-related protein induced by latent Epstein-Barr virus infection in B lymphocytes. J Virol (1996) 70(2): 1143-53.

70. Nishino R, Takano A, Oshita $H$, Ishikawa $N$, Akiyama $H$, Ito $H$, et al. Identification of Epstein-Barr virus-induced gene 3 as a novel serum and tissue biomarker and a therapeutic target for lung cancer. Clin Cancer Res (2011) 17(19):6272-86. doi:10.1158/1078-0432.CCR-11-0060

71. Trojanowska M, LeRoy EC, Eckes B, Krieg T. Pathogenesis of fibrosis: type 1 collagen and the skin. J Mol Med (Berl) (1998) 76(3-4):266-74. doi:10.1007/ s001090050216

72. Li MF, Sun BG, Xiao ZZ, Sun L. First characterization of a teleost EpsteinBarr virus-induced gene 3 (EBI3) reveals a regulatory effect of EBI3 on the innate immune response of peripheral blood leukocytes. Dev Comp Immunol (2013) 41(4):514-22. doi:10.1016/j.dci.2013.07.022 
73. Salvarani C, Cantini F, Hunder GG. Polymyalgia rheumatica and giantcell arteritis. Lancet (2008) 372(9634):234-45. doi:10.1016/S0140-6736(08) 61077-6

74. Moore KW, de Waal Malefyt R, Coffman RL, O'Garra A. Interleukin-10 and the interleukin-10 receptor. Annu Rev Immunol (2001) 19:683-765. doi:10.1146/annurev.immunol.19.1.683

75. Aparicio-Siegmund S, Moll JM, Lokau J, Grusdat M, Schroder J, Plohn S, et al. Recombinant p35 from bacteria can form Interleukin (IL-)12, but not IL-35. PLoS One (2014) 9(9):e107990. doi:10.1371/journal.pone.0107990

76. Lander ES, Linton LM, Birren B, Nusbaum C, Zody MC, Baldwin J, et al. Initial sequencing and analysis of the human genome. Nature (2001) 409(6822): 860-921. doi:10.1038/35057062

77. Venter JC, Adams MD, Myers EW, Li PW, Mural RJ, Sutton GG, et al. The sequence of the human genome. Science (2001) 291(5507):1304-51. doi:10.1126/science. 1058040
Conflict of Interest Statement: The authors declare that the research was conducted in the absence of any commercial or financial relationships that could be construed as a potential conflict of interest.

The reviewer DK and handling Editor declared their shared affiliation, and the handling editor states that the process nevertheless met the standards of a fair and objective review.

Copyright (c) 2016 Hasegawa, Mizoguchi, Chiba, Ohashi, Xu and Yoshimoto. This is an open-access article distributed under the terms of the Creative Commons Attribution License (CC BY). The use, distribution or reproduction in other forums is permitted, provided the original author(s) or licensor are credited and that the original publication in this journal is cited, in accordance with accepted academic practice. No use, distribution or reproduction is permitted which does not comply with these terms. 